\title{
Weighting of topologically different interactions in a model of two-dimensional polymer collapse
}

\author{
Andrea Bedin ${ }^{*}$ and Aleksander L. Owczarek \\ Department of Mathematics and Statistics, The University of Melbourne, 3010, Australia \\ Thomas Prellberg \\ School of Mathematical Sciences, Queen Mary University of London, \\ Mile End Road, London, E1 4NS, United Kingdom
}

\begin{abstract}
We study by computer simulation a recently introduced generalised model of self-interacting self-avoiding trails on the square lattice that distinguishes two topologically different types of selfinteraction: namely crossings where the trail passes across itself and collisions where the lattice path visits the same site without crossing. This model generalises the canonical interacting self-avoiding trail model of polymer collapse which has a strongly divergent specific heat at its transition point. We confirm the recent prediction that the asymmetry does not affect the universality class for a range of asymmetry. Certainly, where the weighting of collisions outweighs that of crossings this is well supported numerically. When crossings are weighted heavily relative to collisions the collapse transition reverts to the canonical $\theta$-point-like behaviour found in interacting self-avoiding walks.
\end{abstract}

PACS numbers: 05.50.+q, 05.70.fh, 61.41.+e

\section{INTRODUCTION}

The collapse transition of a polymer in a dilute solution has been a continuing focus of study in lattice statistical mechanics for decades [1, 2]. This transition describes the change in the scaling of the polymer with length that occurs as the temperature is lowered. At high temperatures the radius of gyration of polymer scales in a way swollen relative to a random walk: this is known as the excluded volume effect. At low temperatures a polymer condenses into dense, usually disordered, globule, with a much smaller radius of gyration. The interest in this phase transition has occurred both because of the motivation of physical systems but also because of the study of integrable cases 3, 4, of lattice models have proved especially fruitful in two dimensions. While the canonical lattice model of the configurations of a polymer in solution has been the self-avoiding walk (SAW), where a random walk on a lattice is not allowed to visit a lattice site more than once, an alternative has been to use bond-avoiding walks, or a self-avoiding trail. A self-avoiding trail (SAT) is a lattice walk configuration where the excluded volume is obtained by preventing the walk from visiting the same bond, rather than the same site, more than once. The model of SAT was used initially to model polymers with loops 5 but has subsequently occurred in integrable loop models in two dimensions [4. A model of collapsing polymers can be constructed starting from self-avoiding trails, known as interacting self-avoiding trails (ISAT). Here energies are associated with multiply-visited sites, and by favouring configurations with many such sites a

\footnotetext{
*Electronic address: abedini@ms.unimelb.edu.au

${ }^{\dagger}$ Electronic address: owczarek@unimelb.edu.au

${ }^{\ddagger}$ Electronic address: t.prellberg@qmul.ac.uk
}

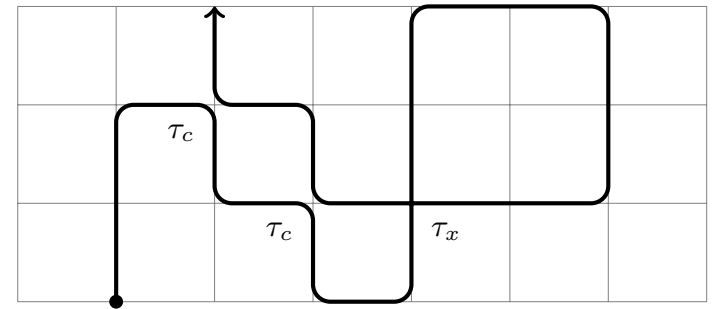

FIG. 1: An example of AISAT configuration with one crossing $\left(m_{x}=1\right)$ and two collisions $\left(m_{c}=2\right)$, associated with the Boltzmann weights $\tau_{x}$ and $\tau_{c}$, respectively. The total number of doubly visited sites is $m_{2}=3$.

collapse transition can be initiated.

Owczarek and Prellberg studied numerically the ISAT collapse on the square lattice by two different approaches [6. 7] and in either case found a strong continuous transition with specific heat exponent $\alpha=0.81(3)$. Recently, on the triangular lattice Doukas et al. 8 found that by changing the weighting of doubly and triply visited sites a first-order transition can ensue or alternatively, depending on the ratio of these weightings, a weaker secondorder transition that mimics the collapse found in the canonical interacting self-avoiding walk (ISAW) model (also known as the $\theta$-point). They also found that the low temperature phase becomes fully dense rather than globular, depending on the choice of parameters.

Recently, Foster 9] generalised the ISAT model on the square lattice by differentiating the type of doubly visited sites on the square lattice: a doubly visited site can be visited twice with the trail passing through itself, that is crossing, at the site, or alternatively as a result of two bends in the trail so that the trail "touches" or "collides": see Fig. 1.

We shall refer to this model as the asymmetric ISAT 
model (AISAT). The study [9] using transfer matrices and the phenomenological renormalisation group of the AISAT predicted that the universality class of the symmetric case extended to the asymmetric case. However, when crossings sufficiently dominate over collisions the results suggested the appearance of a first-order transition.

In this work we use Monte Carlo simulation to explore this AISAT model and the predictions of Foster [9]. We also explore the low temperature phase of the model and find that it is fully dense for a range of asymmetry including the symmetric case.

\section{ISAT}

The model of interacting trails on the square lattice is defined as follows. Consider the ensemble $\mathcal{T}_{n}$ of selfavoiding trails (SAT) of length $n$, that is, of all lattice paths of $n$ steps that can be formed on the square lattice such that they never visit the same bond more than once. Given a SAT $\psi_{n} \in \mathcal{T}_{n}$, we associate an energy $-\varepsilon_{t}$ with each doubly visited site and denote by $m_{j}\left(\psi_{n}\right)$ the number sites visited $j$ times by $\psi_{n}$. We have $n=m_{1}+2 m_{2}$. The probability of $\psi_{n}$ is given by

$$
\frac{e^{\beta \varepsilon_{t} m_{2}\left(\psi_{n}\right)}}{Z_{n}^{I S A T}(T)}
$$

where we define the Boltzmann weight $\omega_{t}=\exp \left(\beta \varepsilon_{t}\right)$ and $\beta$ is the inverse temperature $1 / k_{B} T$. The partition function of the ISAT model is given by

$$
Z_{n}^{I S A T}(T)=\sum_{\psi_{n} \in \mathcal{T}_{n}} \omega_{t}^{m_{2}\left(\psi_{n}\right)} .
$$

The finite-length reduced free energy is

$$
\kappa_{n}(T)=\frac{1}{n} \log Z_{n}(T)
$$

and the thermodynamic limit is obtained by taking the limit of large $n$, i.e.,

$$
\kappa(T)=\lim _{n \rightarrow \infty} \kappa_{n}(T) .
$$

It is expected that there is a collapse phase transition at a temperature $T_{c}$ characterized by a non-analyticity in $\kappa(T)$.

The collapse transition can be characterized via a change in the scaling of the size of the polymer with temperature. It is expected that some measure of the size, such as the radius of gyration or the mean squared distance of a monomer from the end points, $R_{n}^{2}(T)$, scales at fixed temperature as

$$
R_{n}^{2}(T) \sim A n^{2 \nu}
$$

with some exponent $\nu$. At high temperatures the polymer is swollen and in two dimensions it is accepted that $\nu=3 / 4[3]$. At low temperatures the polymer becomes dense in space, though not space filling, and the exponent is $\nu=1 / 2$. The collapse phase transition is expected to take place at some temperature $T_{c}$. If the transition is second-order, the scaling at $T_{c}$ of the size is intermediate between the high and low temperature forms. In the thermodynamic limit there is expected to be a singularity in the free energy, which can be seen in its second derivative (the specific heat). Denoting the (intensive) finite length specific heat per monomer by $c_{n}(T)$, the thermodynamic limit is given by the long length limit as

$$
C(T)=\lim _{n \rightarrow \infty} c_{n}(T) .
$$

One expects that the singular part of the specific heat behaves as

$$
C(T) \sim B\left|T_{c}-T\right|^{-\alpha},
$$

where $\alpha<1$ for a second-order phase transition. The singular part of the thermodynamic limit internal energy behaves as

$$
U(T) \sim B\left|T_{c}-T\right|^{1-\alpha}
$$

if the transition is second-order, and there is a jump in the internal energy if the transition is first-order (an effective value of $\alpha=1$ ).

Moreover one expects crossover scaling forms [10] to apply around this temperature, so that

$$
c_{n}(T) \sim n^{\alpha \phi} \mathcal{C}\left(\left(T-T_{c}\right) n^{\phi}\right)
$$

with $0<\phi<1$ if the transition is second-order, and

$$
c_{n}(T) \sim n \mathcal{C}\left(\left(T-T_{c}\right) n\right)
$$

if the transition is first-order. From [10] we point out that the exponents $\alpha$ and $\phi$ are related via

$$
2-\alpha=\frac{1}{\phi} .
$$

Important for numerical estimation is the use of equation II.9 at the peak value of the specific heat given by $y^{\text {peak }}=\left(T-T_{c}\right) n^{\phi}$ so that

$$
c_{n}^{\text {peak }}(T) \sim \mathcal{C}^{\text {peak }} n^{\alpha \phi}
$$

where $\mathcal{C}^{\text {peak }}=\mathcal{C}\left(y^{\text {peak }}\right)$ is a constant.

A previous study [7] of ISAT model on the square lattice has shown that there is a collapse transition with a strongly divergent specific heat, with

$$
\alpha \phi=0.68(5)
$$

and so the individual exponents have been estimated as

$$
\phi=0.84(3) \quad \text { and } \quad \alpha=0.81(3) .
$$

At $T=T_{c}$ it was predicted [6] that

$$
R_{n}^{2}(T) \sim A n(\log n)^{2} .
$$




\section{ASYMMETRIC ISAT MODEL}

The Asymmetric ISAT (AISAT) model can be defined as follows. Consider the set of bond-avoiding paths $\mathcal{T}_{n}$ as defined in the previous section. Given a SAT $\psi_{n} \in \mathcal{T}_{n}$, we associate an energy with each doubly visited site, as in ISAT, but we make a distinction between whether the trail crosses itself or not. We will call the former crossings and the latter collisions with associated energies $-\varepsilon_{x}$ and $-\varepsilon_{c}$, respectively. For each configuration $\psi_{n} \in \mathcal{T}_{n}$ we count the number $m_{x}\left(\psi_{n}\right)$ of crossings and $m_{c}\left(\psi_{n}\right)$ of collisions, see Fig. 1. Note that the total number of doubly visited sites is $m_{2}=m_{c}+m_{x}$. We associate with each configuration a Boltzmann weight $\tau_{x}^{m_{x}\left(\psi_{n}\right)} \tau_{c}^{m_{c}\left(\psi_{n}\right)}$ where $\tau_{x}=\exp \left(\beta \varepsilon_{x}\right), \tau_{c}=\exp \left(\beta \varepsilon_{c}\right)$ and $\beta$ is the inverse temperature $1 / k_{B} T$. The partition function of the AISAT model is the given by

$$
Z_{n}\left(\tau_{x}, \tau_{c}\right)=\sum_{\psi_{n} \in \mathcal{T}_{n}} \tau_{x}^{m_{x}\left(\psi_{n}\right)} \tau_{c}^{m_{c}\left(\psi_{n}\right)}
$$

The probability of a configuration $\psi_{n}$ is then

$$
p\left(\psi_{n} ; \tau_{x}, \tau_{c}\right)=\frac{\tau_{x}^{m_{x}\left(\psi_{n}\right)} \tau_{c}^{m_{c}\left(\psi_{n}\right)}}{Z_{n}\left(\tau_{x}, \tau_{c}\right)} .
$$

In line with Foster 9 let us define the variables

$$
x=\frac{\tau_{x}}{\tau_{c}}
$$

and

$$
r=\frac{x}{1+x}=\frac{\tau_{x}}{\tau_{x}+\tau_{c}} .
$$

When we set $\tau_{x}=\tau_{c}(x=1, r=1 / 2)$ the model reduces to the ISAT model, in which crossings and collisions are given the same weight. On the other end, if we set $\tau_{x}=0$ $(x=r=0)$ configurations with crossings are excluded and our model reduces to the VISAW model [11.

The average of any quantity $Q$ over the ensemble set of path $\mathcal{T}_{n}$ is given generically by

$$
\langle Q\rangle\left(n ; \tau_{x}, \tau_{c}\right)=\sum_{\psi_{n} \in \mathcal{T}_{n}} Q\left(\psi_{n}\right) p\left(\psi_{n} ; \tau_{x}, \tau_{c}\right)
$$

In particular, we can define the average number of crossings and collisions per site and their respective fluctuations as

$$
\begin{array}{ll}
u_{x}=\frac{\left\langle m_{x}\right\rangle}{n}, & c_{x}=\frac{\left\langle m_{x}^{2}\right\rangle-\left\langle m_{x}\right\rangle^{2}}{n}, \\
u_{c}=\frac{\left\langle m_{c}\right\rangle}{n}, & c_{c}=\frac{\left\langle m_{c}^{2}\right\rangle-\left\langle m_{c}\right\rangle^{2}}{n} .
\end{array}
$$

An important quantity for what follows is the proportion of the sites on the trail that are at lattice sites which are not doubly occupied:

$$
p_{n}=1-\frac{2}{n}\left(\left\langle m_{c}\right\rangle+\left\langle m_{x}\right\rangle\right)
$$

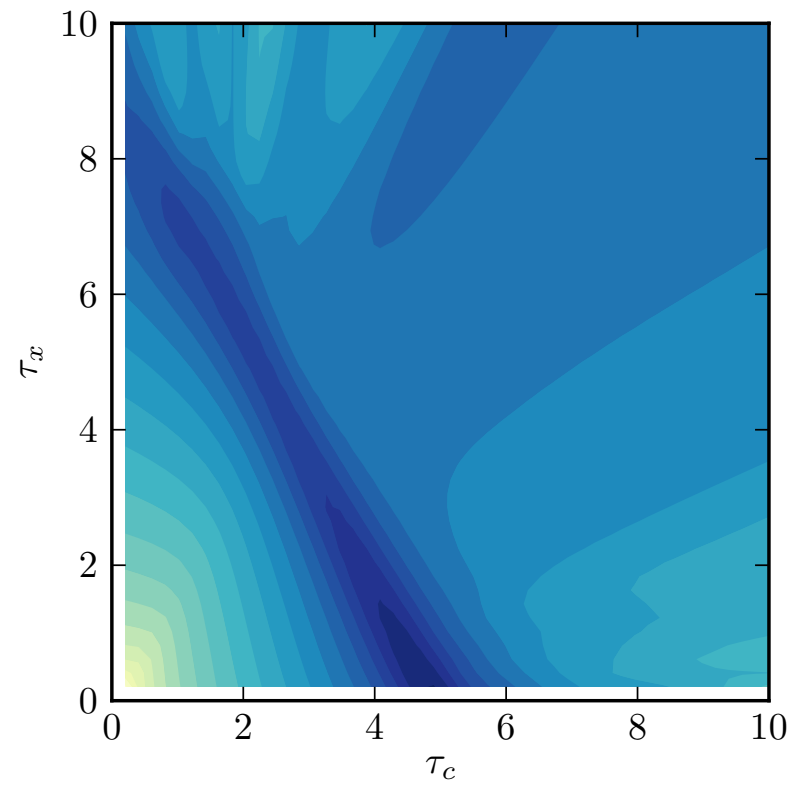

FIG. 2: Density plot of the logarithm of the largest eigenvalue $\lambda_{\max }$ of the matrix of second derivatives of the free energy with respect to $\tau_{x}$ and $\tau_{c}$ at length 500 .

Foster 9 predicted that the universality class of symmetric ISAT at $r=1 / 2$ extends to other values of $r$ and further that there may be a change to a first-order transition for large values of $r$.

\section{NUMERICAL RESULTS}

We began by simulating the full two parameter space by using the flatPERM algorithm [12. FlatPERM outputs an estimate $W_{n, \mathbf{k}}$ of the total weight of the walks of length $n$ at fixed values of some vector of quantities $\mathbf{k}=\left(k_{1}, k_{2}, \ldots, k_{\ell}\right)$. From the total weight one can access physical quantities over a broad range of temperatures through a simple weighted average, e.g.

$$
\langle\mathcal{O}\rangle_{n}(\tau)=\frac{\sum_{\mathbf{k}} \mathcal{O}_{n, \mathbf{k}}\left(\prod_{j} \tau_{j}^{k_{j}}\right) W_{n, \mathbf{k}}}{\sum_{\mathbf{k}}\left(\prod_{j} \tau_{j}^{k_{j}}\right) W_{n, \mathbf{k}}} .
$$

The quantities $k_{j}$ may be any subset of the physical parameters of the model. In our case we begin by using $k_{1}=m_{x}$ and $k_{2}=m_{c}$.

We have simulated AISAT using the full two-parameter flatPERM algorithm up to length $n=500$, with $10^{5}$ iterations, collecting $1.2 \cdot 10^{10}$ samples at the maximum length. Following [12, we also measured the number of samples adjusted by the number of their independent growth steps ("effective samples") $S^{\text {eff }} \simeq 3.9 \cdot 10^{8}$ at the maximum length. To obtain a landscape of possible phase transitions we plot the largest eigenvalue of 


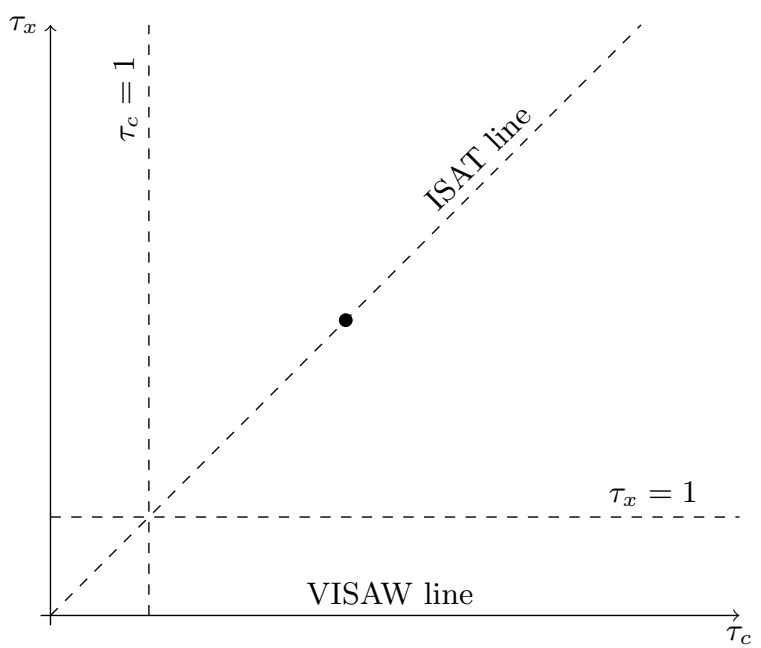

FIG. 3: Schematic diagram of the AISAT parameter space. The black dot on the diagonal line indicates the ISAT critical point. We have simulated along the dashed lines, as well as the VISAW line.

the matrix of second derivatives of the free energy with respect to $\tau_{x}$ and $\tau_{c}$ at length $n=500$ in Fig. 2.

We notice that there is a strong peak in the fluctuations running in a line from $\tau_{c} \approx 5$ when $\tau_{x}=0(r=0)$ to $\tau_{x} \approx 8$ when $\tau_{c}=0(r=1)$. It is interesting to observe that the peak in the fluctuations becomes weaker as $r$ increases from 0 to 1.

Next, we have simulated four different one parameter slices of the AISAT model all up to length $n=1024$. Their location, as indicated in Fig. 3 is as follows.

- $\tau_{x}=0$ (the VISAW model). With $S \simeq 1.8 \cdot 10^{7}$ iterations, collecting $4.5 \cdot 10^{10}$ samples at the maximum length (corresponding to $S^{\text {eff }} \simeq 5.2 \cdot 10^{8}$ ).

- $\tau_{x}=1$ (the colliding model). With $7.8 \cdot 10^{6}$ iterations, collecting $2.6 \cdot 10^{10}$ samples at the maximum length (corresponding to $S^{e f f} \simeq 4.1 \cdot 10^{8}$ ).

- $\tau_{x}=\tau_{c}$ (the symmetric ISAT model). With $S \simeq$ $4 \cdot 10^{6}$ iterations, collecting $7.6 \cdot 10^{9}$ samples at the maximum length (corresponding to $S^{e f f} \simeq 5.8$. $\left.10^{8}\right)$.

- $\tau_{c}=1$ (the crossing model). With $S \simeq 7.7 \cdot 10^{6}$ iterations, collecting $2.7 \cdot 10^{10}$ samples at the maximum length (corresponding to $S^{e f f} \simeq 2.7 \cdot 10^{8}$ ).

\section{A. Specfic heat}

We have begun by analysing the scaling of the specific heat by calculating the location of its peak $\tau_{n}^{p}=$ $\arg \max _{\tau} c_{n}(\tau)$ and thereby evaluating $c_{n}^{p}=c_{n}\left(\tau_{n}^{p}\right)$. In Fig. 4. we plot the peak values of the specific heat for the four models we have simulated. The exponent associated with the peak of the specific heat, see equation (II.12), is $\alpha \phi$ if the transition is second order. For the symmetric ISAT model, we estimated $\alpha \phi \approx 0.64$, which is a little less that our previous estimate (equation (II.13)) based upon much longer length trails. We find for the VISAW $(\alpha \phi \approx 0.69)$ and the colliding model $(\alpha \phi \approx 0.63)$ exponent estimates compatible with that of symmetric ISAT collapse at this length. This is a crucial result as it confirms the prediction of Foster 9 that the universality class of ISAT extends from $r=1 / 2$ to other values of $r$.

We see that an attempted estimate of $\alpha \phi$ for the crossing model over the full range of lengths is compromised by poor simulation results. Nevertheless there is clear curvature in the data and the estimate decreases with length. Using data from around length 400 onwards gives an estimate of 0.08 . Given that this estimate would most likely decrease even further with increasing length, it is likely that the data is compatible with the behaviour of the $\theta$-point interacting SAW collapse transition; this has a negative value of the exponent $\alpha$.

Therefore our data indicates that the ISAT universality class extends to a range values of the parameter $r$ measuring the asymmetry, as conjectured by Foster [9. Intriguingly, rather than becoming first order for $r$ near one as also conjectured in [9], the transition becomes weaker and potentially $\theta$-like as $r$ increases towards one.

\section{B. Study of the low temperature region}

To further investigate the proposition that changing the asymmetry over a range around $r$ does not affect the nature of the collapse, except potentially near $r$ near 1 , we examine the low temperature phase. This approach proved fruitful in the case of triangular lattice extended ISAT 8 where the low temperature phase could be either globular or fully dense. The fully dense low temperature phase seems associated with either the ISAT universality class or a first-order transition, while the globular phase is associated with the much weaker (non-divergent specific heat) ISAW ( $\theta$-point) collapse transition universality class. Here we present evidence that for the square lattice symmetric ISAT, colliding and VISAW models the low-temperature phase is maximally dense, and that the density jumps discontinuously at the critical point. On the other hand for the crossing model the low temperature phase seems not to be fully dense. This would be compatible with the conjecture that the collapse transition for the crossing model is $\theta$-point-like from a swollen phase at high temperatures to a globular phase at low temperatures.

We have considered two different approaches to measuring the density. The first is indirect by measuring the proportion of sites of the lattice visited only once by the trail, and the second is using the radius of gyration to estimate the internal density of the polymer. Both lead to the same conclusions. 


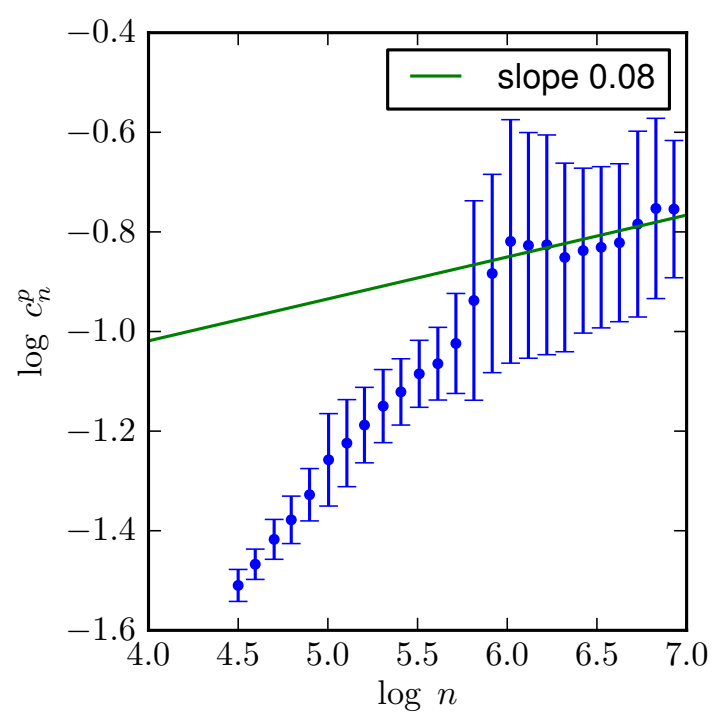

(a)The crossing model, $\tau_{c}=1$

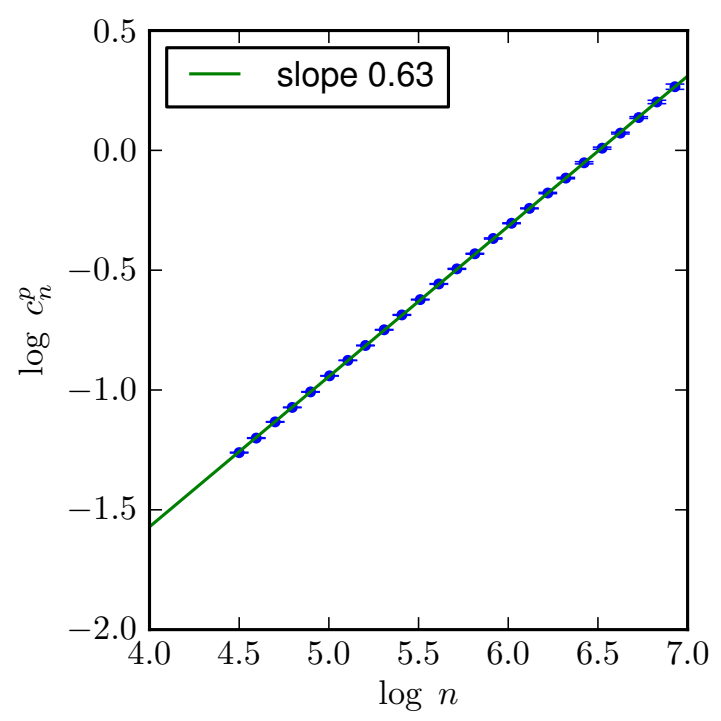

(c)The colliding model, $\tau_{x}=1$

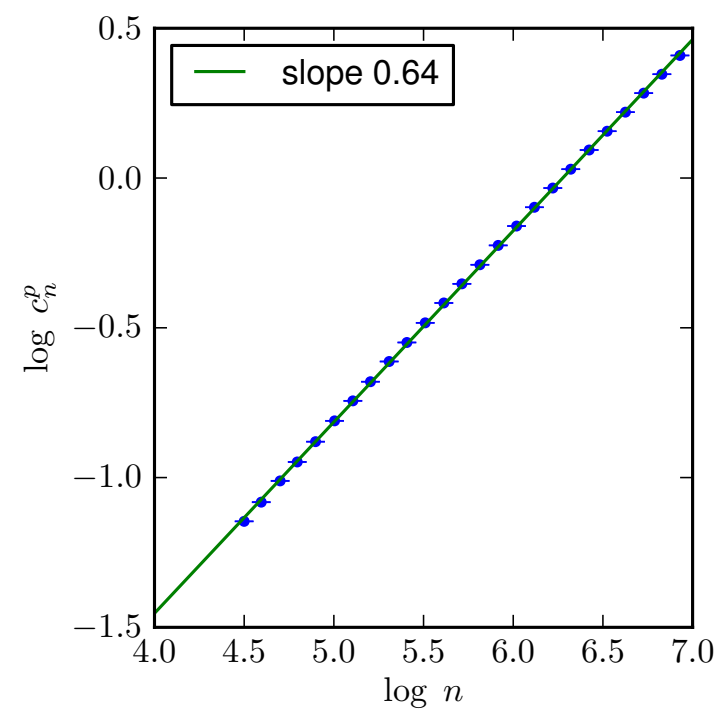

(b)The symmetric ISAT model, $\tau_{c}=\tau_{x}$

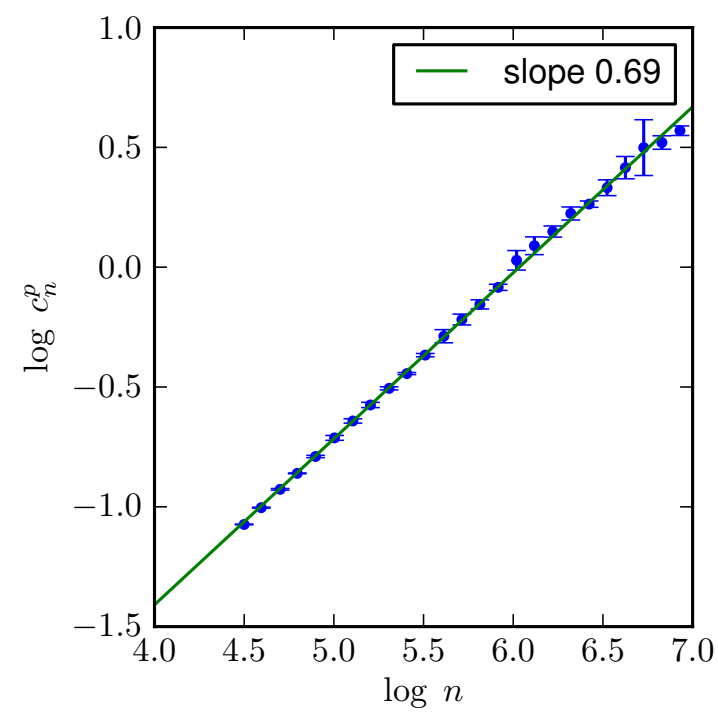

(d)The VISAW model, $\tau_{x}=0$

FIG. 4: Double-logarithmic plots of the peak value of the specific heat against length for the four models defined at the beginning of Section 4. For three of the models the specific heat diverges strongly, while for the Crossing model the behaviour of the specific heat is markedly different: the data is consistent with a converging specific heat.

\section{Proportion of singly visited sites $p_{n}$}

Following the analysis in 8 we first measured the proportion $p_{n}$ of sites of the lattice visited only once by the trail. This provides a useful method for considering how dense our configurations are on average since an asymptotic value of zero would imply that effectively all the sites occupied by the trail are doubly occupied. Double occupation of lattice sites implies that the surrounding edges of the lattice are all occupied with bonds of the trail: hence the lattice is filled by the trail as the trail increases in length. At high temperatures it is easy to see that $p_{n}$ approaches a finite strictly positive value in the thermodynamic limit: this is connected with the swollen nature of the polymer as seen in the radius of gyration scaling. One would expect on physical grounds that $p_{\infty}(T)$ would be a monotonically increasing function of temperature $T$. The question that arises is whether at low temperatures this value is zero and what kind of singularity occurs in $p_{\infty}(T)$ at the collapse transition. 


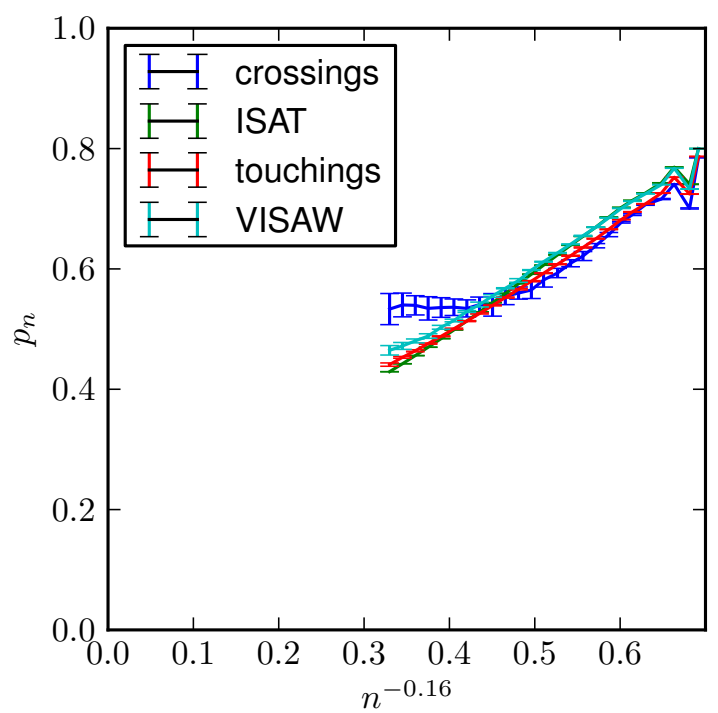

(a)Critical temperature

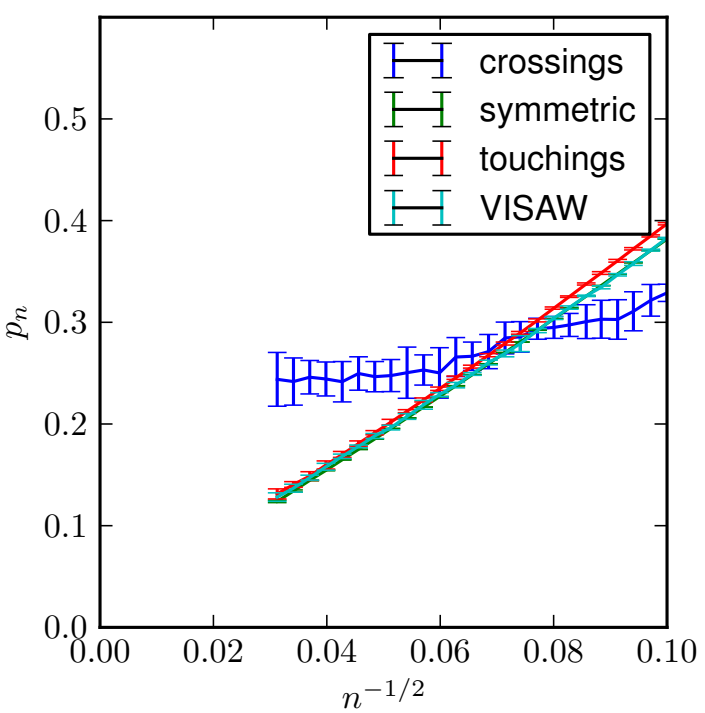

(b)Low temperature

FIG. 5: Plots of $p_{n}$, the proportion of steps visiting the same site once, at a low temperature (on the left) and at the critical temperature (on the right). The scale $n^{-1 / 2}$ chosen is the natural low temperature scale. The chosen temperatures for the plot on the left are $\tau=20$ (crossings), $\tau=5$ (ISAT), $\tau=7$ (colliding), $\tau=8$ (VISAW). At the critical temperature, the quantity $p_{n}$ is plotted against the crossover exponent obtained in [7] $(1-\alpha) \phi \simeq 0.16$.

It is worth considering first what happens at the collapse point itself in the symmetric ISAT model. Thanks to the mapping between critical ISAT and Kinetic Growth Trails we know that critical limiting value for $p_{n}$ is exactly $1 / 5$ [7. The argument goes as follows: consider the case when a trail has formed a large $n$-step loop which occupies $m_{1}+m_{2}=M$ lattice sites (this is always the case as trails do not trap). Any site of this loop could have been the starting point. In order for this site to be visited only once, the loop must have closed at the first return visit, which occurs with a probability of $1 / 3$. Therefore we find for large loops $m_{1} / M \rightarrow 1 / 3$, from whence it follows $p_{n}=m_{1} / n \rightarrow 1 / 5$. In Fig. 5 (a), we plotted $p_{n}$ against $n^{-0.16}$ at the estimated critical temperatures of our four sub-models. We have used $(1-\alpha) \phi \simeq 0.16$ as the appropriate correction to scaling exponent from the estimated exponents for the symmetric ISAT model with $\alpha \phi \approx 0.68$. For the symmetric ISAT, colliding and VISAW model this choice seems appropriate; in each case the estimated value of $p_{\infty}$ is close to 0.2 . While the correction to scaling exponent may not be appropriate for the crossing model it is clear that any estimate of $p_{\infty}$ is greater than 0.2 .

For low temperatures, as discussed above, if the trail fills the lattice asymptotically in a fully dense phase the portion of monomers of the trail not involved with doubly-visited sites of the lattice should tend to zero as $n \rightarrow \infty$. In Fig. 5 (b), we plotted $p_{n}$ against $n^{-1 / 2}$ for our four sub-models. The plots suggest that for the symmetric ISAT, colliding and VISAW model, $p_{n} \rightarrow 0$ as $n \rightarrow \infty$ in the low-temperature region, which implies a maximally-dense phase: our extrapolation estimates for $p_{\infty}$ have error bars encompassing zero. The crossing model seems to show a different behavior: our data suggest that $p_{n}$ tends to a non-zero value (around 0.25 )

These results form a clear picture in which the quantity $p_{\infty}$ jumps discontinuously to zero at the critical temperature for the symmetric ISAT, colliding and VISAW models. In contrast, for the crossing model $p_{\infty}$ remains non-zero for all temperatures. In Fig. 6, we have plotted the quantity $p_{n}$ at different length scales as a function of the temperature. The plots again suggest a common discontinuous behavior for the last three models, while the crossing model seems to show a continuous transition in line with the extrapolations described in the previous paragraph.

These findings support the result of the specific heat analysis, namely that the universality classes of the symmetric ISAT, colliding and VISAW models are likely to be the same, while the crossing model is clearly in a distinct universality class.

\section{Density}

A more direct way of measuring of the density is to consider the quantity $\rho=n / R^{2}$ where $R$ is the radius of gyration of the polymer.

For any AISAT in the high temperature phase we expect $R_{n}^{2} \sim n^{2 \nu}$ with $\nu=3 / 4$, as for SAWs and SAT, and 


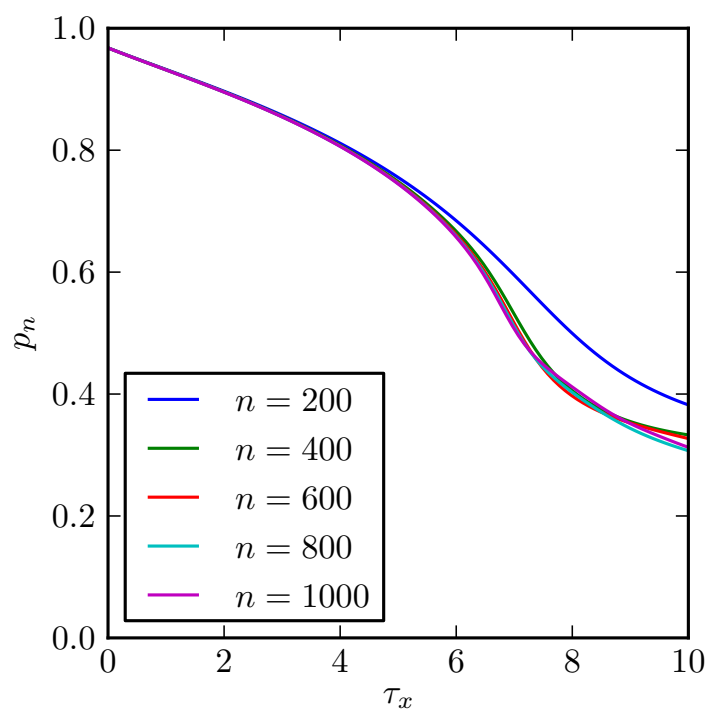

(a)The crossing model, $\tau_{c}=1$

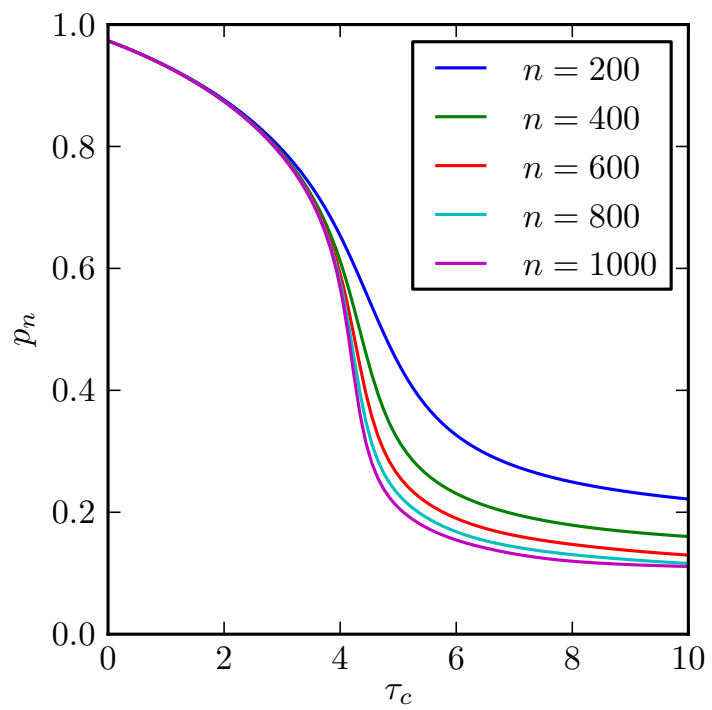

(c)The colliding model, $\tau_{x}=1$

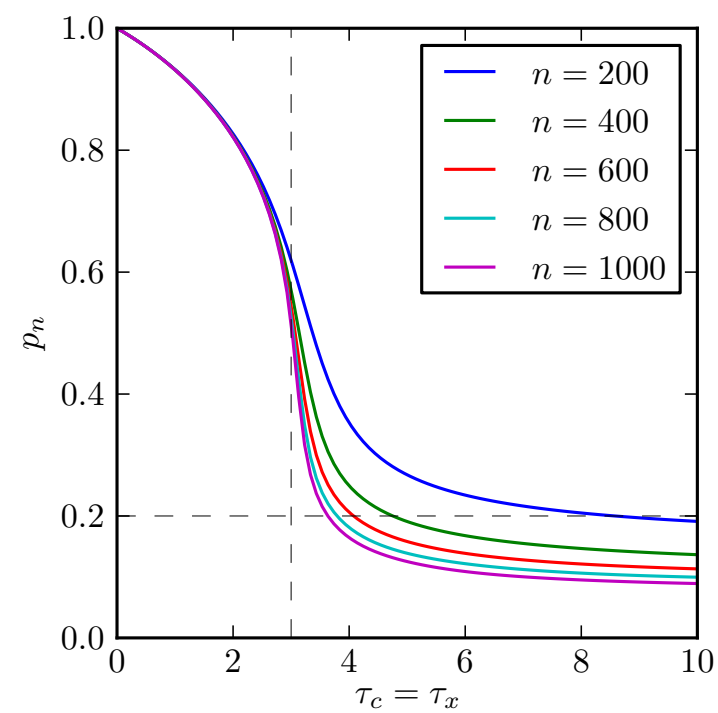

(b)The symmetric ISAT model, $\tau_{c}=\tau_{x}$

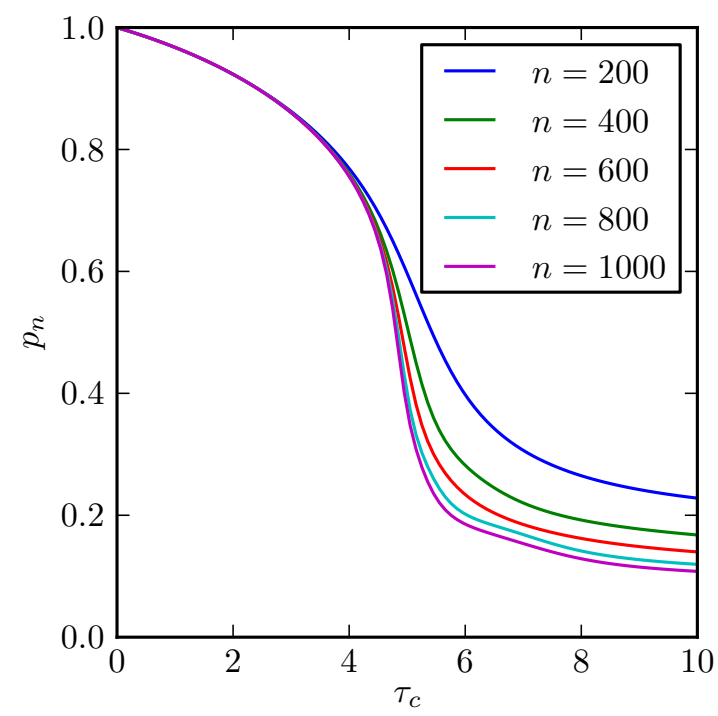

(d)The VISAW model, $\tau_{x}=0$

FIG. 6: Density measured as the fraction $p_{n}$ of sites visited only once as function of the length scale $n$ and of the temperature, for the four models defined at the beginning of Section 4. In (b) the dashed lines indicate the critical temperature and the corresponding value of $p_{n}$. For three of the models $p_{n}$ tends to zero above a critical value of $\tau$, while for the Crossing model $p_{n}$ converges to a non-zero value for all values of $\tau$.

therefore $\rho_{n}=n / R_{n}^{2} \rightarrow 0$. From [ $[$ ] we know that at critical point of the symmetric model $R_{n} \sim n^{1 / 2} \log n$, so the density $\rho$ is zero also at the critical point. For all our models in the collapsed phase we expect that $\rho_{\infty}(T)$ is non-zero at low temperatures. The natural question that arises is whether $\rho_{\infty}(T)$ increases from zero at $T=T_{c}$ as the temperature is lowered in a continuous fashion, or whether it jumps discontinuously to a fixed maximum value at soon as the temperature is smaller than $T_{c}$, being then constant for all $T<T_{c}$.

If we take our results for $p_{n}$ as a guide we would expect that for the ISAT, the colliding and VISAW models $\rho_{\infty}(T)$ the density would jump discontinuously on decreasing $T$ below $T_{c}$ while for the crossing model $\rho_{\infty}(T)$ would increases continuously from zero as $T$ is lowered through $T_{c}$.

In Fig. 7 we plotted $\rho_{n}=n / R_{n}^{2}$ as a function of the temperature. In the symmetric ISAT, colliding and VI- 


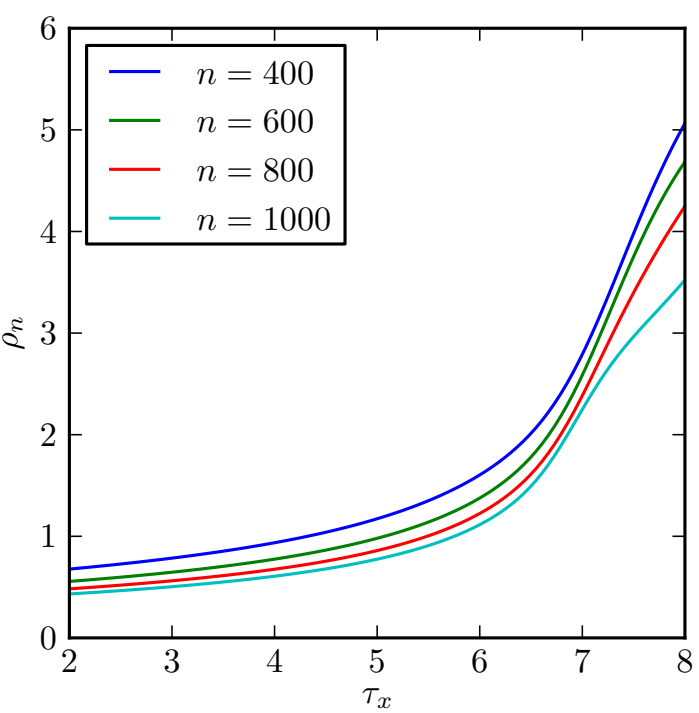

(a)The crossing model, $\tau_{c}=1$

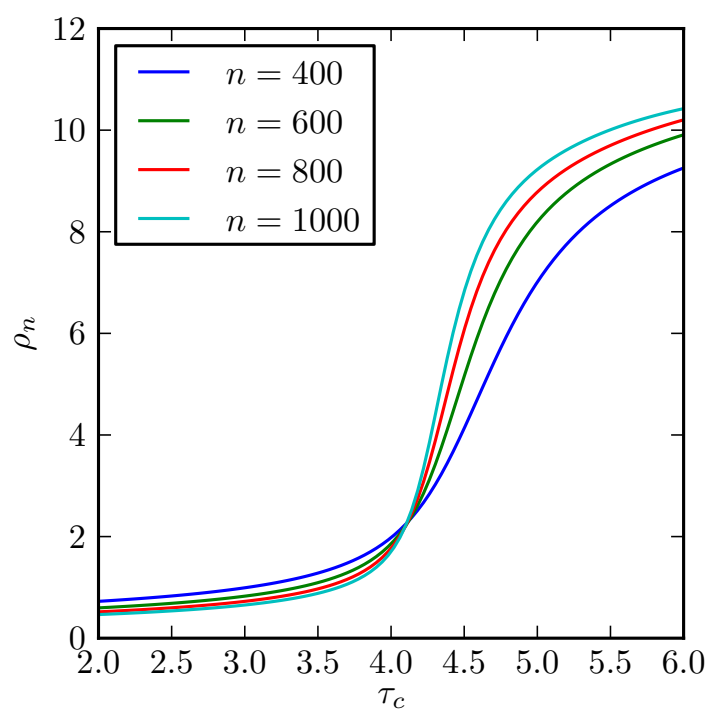

(c)The colliding model, $\tau_{x}=1$

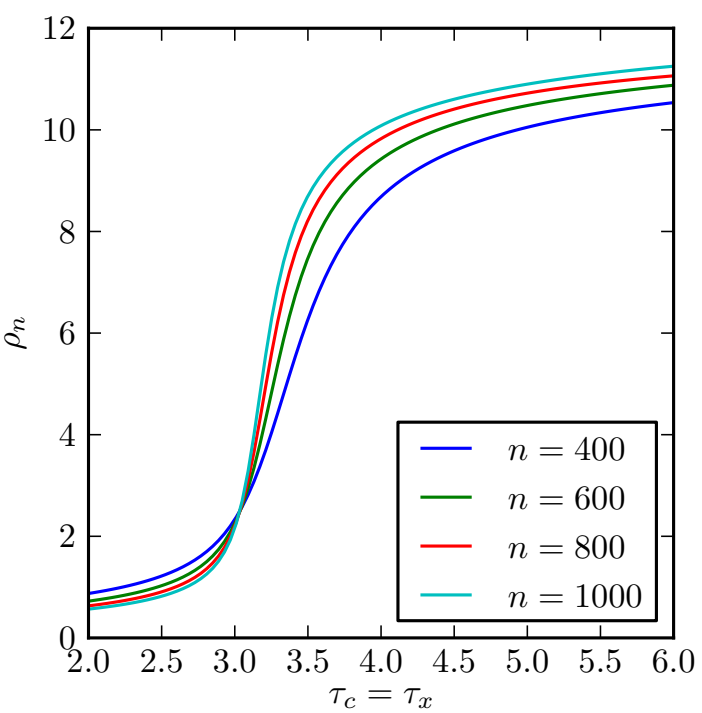

(b)The symmetric ISAT model, $\tau_{c}=\tau_{x}$

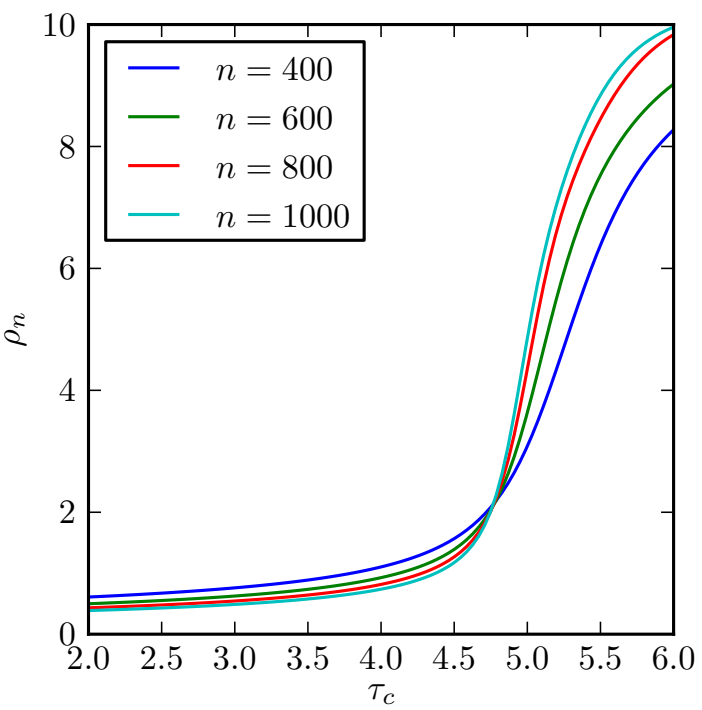

(d)The VISAW model, $\tau_{x}=0$

FIG. 7: Plot of the density $\rho_{n}=n / R_{n}^{2}$ as function of the temperature, for the four models defined at the beginning of Section 4. For three of the models there is a clear crossing point such that for small values of $\tau$ the value of the density decreases with length, while for large values of $\tau$ the value of the density increases with length. For the Crossing model no such crossing point exists, and the density decreases with length for all values of $\tau$.

SAW models the density plotted for different lengths cross at a Boltzmann weight close to the expected collapse transition point, increasing to the right and decreasing to the left. As argued above, the curves converge to zero for small Boltzmann weights (high temperatures) and are consistent with an increase to a temperatureindependent constant for large Boltzmann weights (low temperatures). This is consistent with the behaviour of $p_{n}$ discussed previously. The crossing model seems to have a different behavior. Consistent with a varying limiting value of $p_{n}$, the limiting value of the density $\rho_{n}$ does not approach a temperature-independent constant, either. 


\section{CONCLUSIONS}

We have studied a generalised model of interacting selfavoiding trails (ISAT) on the square lattice as proposed by Foster [9] where the weight associated with crossingtype interactions $\left(\tau_{x}\right)$ and collision-type interactions $\left(\tau_{c}\right)$ may differ.

From our analysis, we can confirm the conjecture in [9] that the ISAT universality class extends over a region of asymmetry around $\tau_{x}=\tau_{c}$. We can conclude that this region extends down to $\tau_{x}=0$, which is also known as the VISAW model, and seems extends to some larger $\tau_{x}>\tau_{c}$. Our simulation results for $\tau_{c}=1$ are compromised numerically by poor convergence: in fact at small lengths we see some evidence of multiply peaked probability distributions but these seem to become unimodal at larger lengths. Importantly, the peak of the specific heat seems to decrease on increasing $x=\tau_{x} / \tau_{c}$. Indeed the specific heat of the $\tau_{c}=1$ model diverges with an effective exponent that is much smaller (albeit with large error bars) than the ISAT universality class would predict and, in fact, the specific heat may not diverge: we would expect a more strongly diverging specific heat if a first-order transition occurs. Supporting these conclusions is our investigation of the low temperature phase for different asymmetry. For the symmetric ISAT and the VISAW model the low temperature phase seems to be fully dense as it is in the extended triangular lattice model in certain regimes. Also, in agreement with our tentative prediction for the $\tau_{c}=1$ case is the evidence that here the low temperature phase is no longer fully dense, which implies a globular ISAW-like low temperature phase. Putting this information together leads us to predict a $\theta$-point-like collapse as occurs in interacting self-avoiding walks for $x$ sufficiently large. Further numerical work is clearly needed to pin down the large $x$ behaviour of the AISAT model, including where the change of universality class occurs. Finally, we point out that our evidence that the low temperature phase is fully dense for symmetric ISAT implies that there is a first-order characteristic of this transition, as predicted by Foster 9], even though there is no latent heat.

\section{Acknowledgements}

Financial support from the Australian Research Council via its support for the Centre of Excellence for Mathematics and Statistics of Complex Systems and through its Discovery program is gratefully acknowledged by the authors. A L Owczarek thanks the School of Mathematical Sciences, Queen Mary, University of London for hospitality.
[1] P.-G. de Gennes, J. Physique Lett. 36, L55 (1975).

[2] P.-G. de Gennes, Scaling Concepts in Polymer Physics, Cornell University Press, Ithaca, 1979.

[3] B. Nienhuis, Phys. Rev. Lett. 49, 1062 (1982).

[4] S. O. Warnaar, M. T. Batchelor, and B. Nienhuis, J. Phys. A. 25, 3077 (1992).

[5] A. Malakis, Physica 84, 256 (1976).

[6] A. L. Owczarek and T. Prellberg, J. Stat. Phys. 79, 951 (1995).

[7] A. L. Owczarek and T. Prellberg, Physica A 373, 433 (2007).
[8] J. Doukas, A. L. Owczarek, and T.Prellberg, Phys. Rev. E 82, 031103 (12pp) (2010).

[9] D. P. Foster, Phys. Rev. E 84, 032102 (2011).

[10] R. Brak, A. L. Owczarek, and T. Prellberg, J. Phys. A. 26, 4565 (1993).

[11] H. W. J. Blöte, M. T. Batchelor, and B. Nienhuis, Physica A: Statistical Mechanics and its Applications 251, 95 (1998).

[12] T. Prellberg and J. Krawczyk, Phys. Rev. Lett. 92, 120602 (2004). 